\title{
Infrared light-absorbing gold/gold sulfide nanoparticles induce cell death in esophageal adenocarcinoma
}

This article was published in the following Dove Press journal:

International Journal of Nanomedicine

14 June 2013

Number of times this article has been viewed

Yan $\mathrm{Li}^{\prime}$

Andre M Gobin ${ }^{2}$

Gerald W Dryden ${ }^{3}$

Xinqin Kang'

Deyi Xiao'

Su Ping $\mathrm{Li}^{\prime}$

Guandong Zhang ${ }^{2}$

Robert CG Martin'

'Department of Surgery,

${ }^{2}$ Department of Bioengineering,

${ }^{3}$ Division of Medicine, University

of Louisville School of Medicine,

Louisville, KY, USA
Correspondence: Yan Li

Department of Surgery, Division

of Surgical Oncology, University

of Louisville School of Medicine,

5 I I S Floyd St, MDR Building,

Room 326A, Louisville,

KY 40202, USA

Tel +I 5028527107

$\mathrm{Fax}+\mathrm{I} 5026293030$

Emailyan.li@louisville.edu
Abstract: Gold nanoparticles and near infrared-absorbing light are each innocuous to tissue but when combined can destroy malignant tissue while leaving healthy tissue unharmed. This study investigated the feasibility of photothermal ablation therapy for esophageal adenocarcinoma using chitosan-coated gold/gold sulfide (CS-GGS) nanoparticles. A rat esophagoduodenal anastomosis model was used for the in vivo ablation study, and three human esophageal cell lines were used to study the response of cancer cells and benign cells to near infrared light after treatment with CS-GGS. The results indicate that both cancerous tissue and cancer cells took up more gold nanoparticles and were completely ablated after exposure to near infrared light. The benign tissue and noncancerous cells showed less uptake of these nanoparticles, and remained viable after exposure to near infrared light. CS-GGS nanoparticles could provide an optimal endoluminal therapeutic option for near infrared light ablation of esophageal cancer.

Keywords: carcinoma, gold nanoparticles, ablation, near infrared, chitosan, drug uptake, cancer

\section{Background}

Esophageal cancer is the seventh most common cause of cancer-related deaths worldwide. ${ }^{1}$ Although squamous cell carcinoma is still the dominant histological type in Europe and Asia, the incidence of adenocarcinoma has been on the increase in the US and Europe, and today accounts for approximately $30 \%$ of all esophageal cancers. $^{2-4}$ The reasons for the increasing incidence are not clear, but hypotheses include population trends in obesity that increase the risk of gastroesophageal reflux, as well as the continuing decline in Helicobacter pylori infection. ${ }^{5}$ Only a subpopulation of patients appears to benefit from treatment, which is generally either surgery or a combination of radiation and chemotherapy for localized tumors. Survival rates are poor for patients with esophageal cancer; patients with advanced metastatic cancer who are treated with palliative chemotherapy have a median survival of less than one year, and the 5-year survival rate of all patients diagnosed is only $14 \%{ }^{6}$

Multimodality therapy remains the optimal treatment strategy for esophageal cancer, and includes surgery, radiotherapy, chemotherapy, and endoscopic therapy. However, limitations of all therapies persist in regard to performance status, comorbidities, intolerance to therapy, and limited local and systemic antineoplastic activity. Neither radiotherapy nor chemotherapy has shown a significant survival advantage. ${ }^{7}$ In addition, esophageal stenosis worsens during the course of radiation therapy, and patients may experience dysphagia and intake disorders, ${ }^{8}$ which can substantially decrease quality of life. Thus, there is a need for novel strategies to improve 
current therapy. The concept of using the combination of a light source and nanoparticles to ablate cancerous tissue without damaging healthy tissue could have a competitive advantage when compared with the current therapeutic strategies for esophageal adenocarcinoma. ${ }^{9}$

Recently, strategies for cancer treatment using polymeric nanoparticles have been developed. Polymeric nanoparticles, which are solid colloidal particles smaller than $1000 \mathrm{~nm}$, can be classified into two main subgroups, ie, nanospheres and nanocapsules. ${ }^{10,11}$ Because nanoparticles have unique characteristics, including a very small particle size, a high surface area, and the possibility of surface modification, they have been attracting much interest for drug delivery purposes during recent years. However, despite much initial promise, their success has so far been limited. Problems related to nanoparticle drug delivery include the limited quantity of nanoparticle preparations available and the toxicity of chemotherapeutic drugs. In addition, targeting specificity remains a key problem. ${ }^{12}$

Light-absorbing metallic nanoparticles designed to have maximal resonance at a particular wavelength can be used in conjunction with a near infrared light source to trigger a photothermal effect in the particles, whereby electronic oscillations at the particle surface are converted into heat. ${ }^{13}$ Several classes of near infrared-absorbing nanoparticle formulations have resulted in particles that are nontoxic to biological tissues and have demonstrated the potential to deliver heat to tumors with minimal damage to healthy surrounding tissues. ${ }^{14}$ These include nanorods, ${ }^{15-18}$ nanocages, ${ }^{11,19}$ nanoshells, ${ }^{20-23}$ and gold/gold sulfide (GGS) nanoparticles. ${ }^{24,25}$ The resulting localized hyperthermia has been shown to induce damage to individual cells, creating a very elegant targeted and potent therapeutic agent. When the nanoparticles get comparatively hot, several effects are produced, including denaturing target cells, triggering bubble nucleation, and imparting a mechanical stress to target cells, the end result of which is cell death. In previous studies, we have demonstrated the photothermal ablation effect of gold nanoparticles targeted to particular cells, ${ }^{26,27}$ and the use of gold sulfide nanoparticles in in vitro and in vivo applications. In the research presented in this paper, GGS nanoparticles were coated with a natural polymer (chitosan) to offer distinctive advantages for biocompatibility. ${ }^{9}$ We used chitosan-coated gold/gold sulfide (CS-GGS) nanoparticles to investigate the effect of photothermal ablation in both esophageal cell lines in vitro and a rat esophageal cancer model in vivo established in our laboratory using surgical esophagoduodenal anastomosis (EDA).

\section{Materials and methods Preparation of CS-GGS nanoparticles}

GGS nanoparticles with controllable near-infrared absorption were prepared by mixing $1.71 \mathrm{mM} \mathrm{HAuCl}_{4}(\mathrm{Au} 49.68 \%$, Alfa Aesar, Ward Hill, MA, USA) with $3 \mathrm{mM} \mathrm{Na}_{2} \mathrm{~S}_{2} \mathrm{O}_{3} 5 \mathrm{H}_{2} \mathrm{O}$ (99.999\%, Sigma-Aldrich, St Louis, MO, USA) solutions. A $\mathrm{Na}_{2} \mathrm{~S}_{2} \mathrm{O}_{3}$ solution was added rapidly to the $\mathrm{HAuCl}_{4}$ solution at a volume ratio to give the desired final peak near infrared wavelength. The size of the GGS nanoparticles observed by transmission electron microscopy was in the range of 30-90 nm, with a hydrodynamic size of approximately $290 \mathrm{~nm}$.

\section{EDA model to induce esophageal cancer}

We established an EDA model to induce stepwise carcinogenetic transformation of esophageal cancer. ${ }^{28,29}$ We have demonstrated that the EDA model is consistent with the clinically relevant pathological changes of esophageal cancer. ${ }^{30,31}$ The EDA surgical procedure was performed as described previously. In brief, 8-week-old Sprague-Dawley rats were anesthetized with $60 \mathrm{mg} / \mathrm{kg}$ of sodium pentobarbital. The gastroesophageal junction was ligated flush with the stomach, and the distal esophagus was transected proximal to the ligature. An enterotomy was made $1 \mathrm{~cm}$ distal to the pylorus on the antimesenteric border. The distal esophagus was anastomosed to the duodenal enterotomy with mucosal-to-mucosal opposition.

\section{Preparation of CS-GGS nanoparticles}

GGS nanoparticles with controllable near infrared absorption were prepared by mixing $1.71 \mathrm{mM} \mathrm{HAuCl}_{4}$ and $3 \mathrm{mM}$ $\mathrm{Na}_{2} \mathrm{~S}_{2} \mathrm{O}_{3} \cdot 5 \mathrm{H}_{2} \mathrm{O}$ solutions.

\section{Murine endoscopy, GGS nanoparticle delivery, and near infrared light exposure}

In this study, we used a murine endoscope ${ }^{32}$ to guide delivery of the gold nanoparticles into the esophageal mucosa, and used a $400 \mu \mathrm{m}$ fiber optic probe for exposure to near infrared light in the EDA rat. Gold nanoparticles were delivered by a 22-gauge aspiration needle through a $2.8 \mathrm{~mm}$ protective sheath with the assistance of a microendoscope. A bare fiber optic probe was inserted into the esophageal lumen through the protective sheath. The light source used for exposure to near infrared light was a $30 \mathrm{~W}$ FAP-I system (Coherent, Santa Clara, CA, USA), with a wavelength of $818 \mathrm{~nm}$. For treatment, a power density of $3 \mathrm{~W} / \mathrm{cm}^{2}$ was used, based on previous studies. ${ }^{22}$ Two procedures were used for exposure to near infrared light, ie, external and internal exposure. The rats were anesthetized for both procedures. For external exposure, the rat was shaved on the abdominal area, with the EDA site 
directly underneath, and 20\% glycerol was applied to the skin to prevent direct injury by near infrared light. The near infrared light probe was placed vertically on the abdominal area directly over the EDA $(10 \mathrm{~cm})$. The near infrared light exposure area was $2 \mathrm{~cm}$ in diameter on the abdominal skin. Internal exposure was performed under microendoscopy, with the near infrared light probe located at the targeted esophageal epithelial tissue. Twenty-four hours after delivery of the CSGGS nanoparticles, exposure to near infrared light was performed at the local targeted tissue, both at high exposure (one minute at $\left.3 \mathrm{~W} / \mathrm{cm}^{2}\right)$ or low exposure $\left(30\right.$ seconds at $\left.1 \mathrm{~W} / \mathrm{cm}^{2}\right)$.

\section{Histopathology}

The entire esophagus was removed and opened longitudinally to examine for evidence of gross morphological changes. Evidence of reflux esophagitis, Barrett's esophagus, and esophageal adenocarcinoma was sought microscopically in the esophageal epithelium as reported elsewhere. ${ }^{33}$ Uptake of the nanoparticles was detected by silver enhancement.

\section{TUNEL assay of ex vivo samples}

In addition to the histology study, a TUNEL assay was used to evaluate cell death in esophageal epithelium closest to the area of near infrared light exposure. An ApopTag ${ }^{\circledR}$ in situ apoptosis detection kit (Intergen Company, Purchase, NY, USA) was used to detect apoptotic cells following a method reported previously. ${ }^{34,35}$

\section{In vitro testing}

Three esophageal cell lines were used to analyze further the response of the esophageal cells to the CS-GGS nanoparticles and near infrared light. The cells used were a human squamous esophageal epithelium cell line (Het-1A), a Barrett's epithelium cell line (BAR-T), and an esophageal adenocarcinoma cell line (OE-19). The three cell types were seeded in 24-well plates at a density of $2 \times 10^{5}$ cells per well for treatment with CS-GGS nanoparticles. Two days after plating, the cells were treated with serum-free medium for 12 hours, and then treated with CS-GGS nanoparticles at a concentration of 1 OD (diluted from a 25 OD stock solution of CS-GGS nanoparticles). The particle concentration at $1 \mathrm{OD}$ is estimated to be $3 \times 10^{11}$ particles per $\mathrm{mL}$ based on scattering theory and geometric/size analysis. Twenty-four hours after application of the nanoparticles, the cells were exposed to near infrared light using an FAP-I laser system at a wavelength of $818 \mathrm{~nm}$ and a power density of high exposure (one minute at $\left.3 \mathrm{~W} / \mathrm{cm}^{2}\right)$ or low exposure $\left(30\right.$ seconds at $\left.1 \mathrm{~W} / \mathrm{cm}^{2}\right)$. Silver staining was used to detect nanoparticle loading in the cells.

\section{Cell viability assay}

To study cell viability after treatment with CS-GGS nanoparticles and near infrared light, we performed a 3-(4, 5-dimethyl-thiazol-2-yl)-2, 5-diphenyltetrazolium bromide (MTT, Sigma-Aldrich) reduction assay according to previous reports. One day after seeding, the cells were treated with either nanoparticles for 12 hours or exposed to near infrared light at either high or low exposure. MTT was added to the cultured cells at $10 \mu \mathrm{L} /$ well $(5 \mathrm{mg} / \mathrm{mL}$ in phosphatebuffered solution) 4 hours before the end of treatment with the nanoparticles and near infrared light. Absorbance was determined spectrophotometrically using a plate reader at a wavelength of $570 \mathrm{~nm}$. Mean optical density values from triplicate wells for each treatment were used as the index of cell viability.

\section{Western blotting}

To study the response of the cells to treatment with nanoparticles and near infrared light, we performed Western blotting to determine the presence of protective proteins (catalase, manganese superoxide dismutase, copper/zinc superoxide dismutase, and heat shock protein 60) along with the cell death effector (caspase-3) in esophageal epithelium.

\section{Statistical analysis}

The Student's $t$-test was performed, assuming unequal variance. The results are expressed as the mean \pm standard deviation. Comparisons were made between groups using analysis of variance. A $P$ value $<0.05$ was considered to be statistically significant.

\section{Results EDA-induced metaplasia and adenocarcinoma in rat esophageal epithelium}

In previous studies, we reported that the endoscopic appearance of EDA esophageal mucosa is characterized by wall thickening, polypoid fold formation, ulceration, and wall hypomotility. In contrast with EDA mucosa, the normal rat esophageal mucosa has a squamous epithelium that looks smooth and white, with blood vessels that are clearly visible beneath the surface. ${ }^{32}$ In this study, we performed murine endoscopy on the EDA rats 4 months after the surgical procedure. At endoscopy, we found morphological changes similar to our previous findings in EDA mucosa. In addition, we found suspicious red-colored 
glandular epithelium in the EDA mucosa (Figure 1A). Before performing photothermal ablation therapy with nanoparticles and laser light, three EDA rats were sacrificed to verify the presence of Barrett's metaplasia and esophageal adenocarcinoma. EDA specimens from three rats were harvested and opened longitudinally to examine for gross abnormalities. The tumor could be seen growing in the EDA site, while the red and texturized mucosa was patched in polypoid folders (Figure 1B). Both the tumor tissue and red mucosa were then isolated and prepared for histological study. Hematoxylin and eosin staining and microscopic imaging demonstrated that the red mucosa and tumor tissue was metaplasia and adenocarcinoma, respectively. On endoscopy, Barrett's metaplasia and adenocarcinoma were found at the anastomotic site but separate from the duodenal epithelium. On microscopy, Barrett's metaplastic epithelium and adenocarcinoma were seen as submucosal ducts with the appearance of goblet cells. The histological changes for Barrett's metaplastic epithelium and adenocarcinoma were consistent with our endoscopic findings. Representative histology of Barrett's esophagus and esophageal adenocarcinoma on staining with hematoxylin and eosin are shown in Figure 1C.

\section{Killing of cells loaded with CS-GGS nanoparticles and exposed to near infrared light in vivo}

After the pilot study for endoscopic determination of metaplasia and adenocarcinoma, we compared the effect of uptake of gold nanoparticles and exposure to near infrared light in EDA and normal esophageal mucosa. CS-GGS nanoparticles were delivered through the endoscope to the lower esophageal lumen, where the appearance of Barrett's metaplasia and adenocarcinoma in the mucosa was highest. Twenty-four hours after delivery of the nanoparticles, the lower esophagus was exposed to near infrared light in the treatment group; the control group received neither nanoparticles nor exposure to near infrared light (Figure 2A). After exposure, the esophageal tissues were harvested to investigate uptake of CS-GGS nanoparticles and the cellular response to subsequent exposure to near infrared light. Hematoxylin and eosin staining and the TUNEL assay were
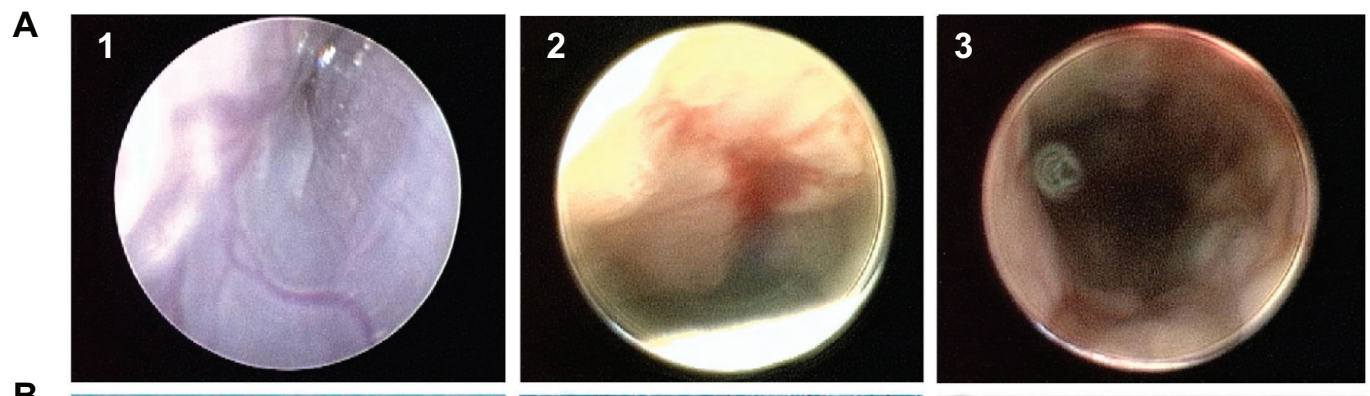

B

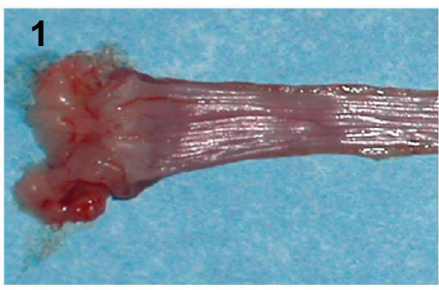

C

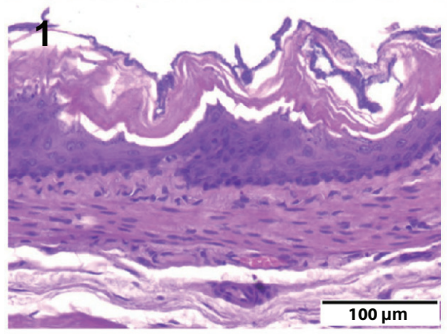

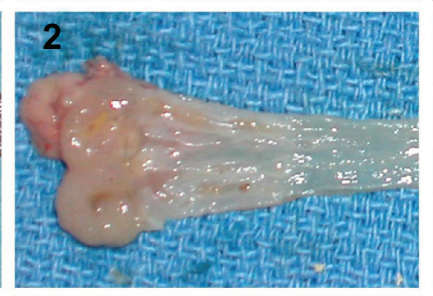

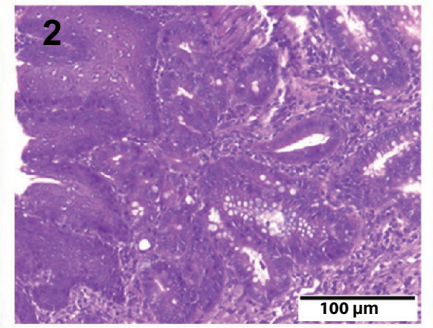

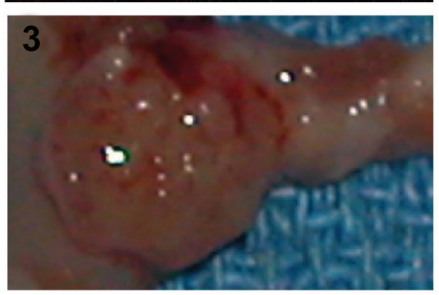

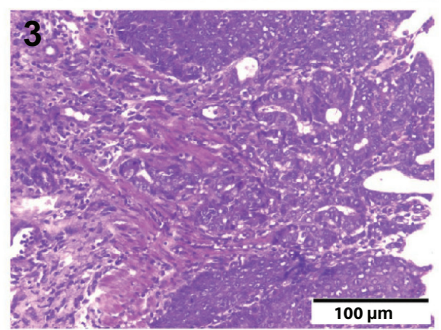

Figure I Endoscopic view, gross anatomy, and histology. (A) Endoscopic view of the esophageal epithelium. (AI) Normal esophageal epithelium; (A2) suspicious glandular epithelium appearing red-colored in the mucosa of the esophagoduodenal anastomosis, which was subsequently demonstrated to be Barrett's metaplasia by histology; and (A3) suspicious glandular epithelium, which was later demonstrated to be esophageal adenocarcinoma by histology. (B) Gross anatomy. (B I) Normal esophageal epithelium; (B2) polypoid folders caused by esophagoduodenal anastomosis, with red mucosal patch in the esophageal epithelium; and (B3) tumor growing in the esophagoduodenal anastomotic site. (C) Histology of esophageal epithelium. (CI) Normal esophageal epithelium; (C2) Barrett's metaplasia; and (C3) esophageal adenocarcinoma. 
A

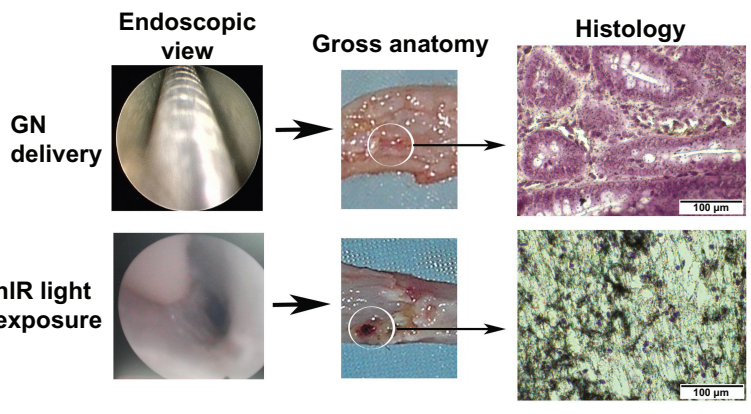

B

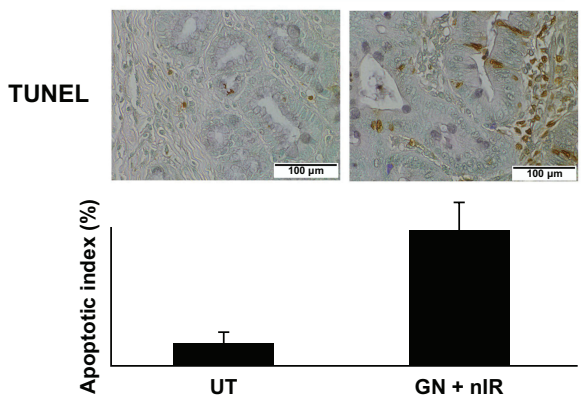

Figure 2 Mucosal ablation in the esophageal lumen as a result of loading with gold nanoparticles and exposure to near infrared light. (A) Delivery of gold nanoparticles and exposure to near infrared light in the esophageal lumen. Gross anatomy and histology in the ablated area is shown. (B) Apoptosis associated with thermal ablation in the adjacent tissue after exposure to near infrared light.

Abbreviations: UT, untreated; GN +nIR, gold nanoparticles treated with infrared light.

used to assess esophageal epithelial cell death and apoptosis. In the slides stained with hematoxylin and eosin, we found that exposure to near infrared light without nanoparticle loading had no effect on the normal esophageal mucosa, metaplastic mucosa, or adenocarcinoma mucosa (data not shown). In contrast, exposure to near infrared light showed cell death when gold nanoparticle loading was evident. Gold nanoparticle loading was demonstrated by histology with silver staining (Figure 2A). As expected, the esophageal mucosa not containing gold nanoparticles remained viable after exposure to near infrared light. To evaluate the tissue adjacent to the region exposed to near infrared light, we did a TUNEL assay on the paraffin-embedded slides, and numerous TUNEL-positive cells were found in the esophageal epithelium of the animals treated with nanoparticles and exposure to near infrared light. However, in control animals which received neither nanoparticles nor near infrared light, the number of TUNEL-positive cells in the esophageal epithelium was much lower. An apoptotic index was obtained by counting the percentage of TUNELpositive epithelial cells, and this was significantly increased in EDA tissue exposed to near infrared light compared with that in the esophageal epithelium of controls $(P<0.05)$. Representative apoptosis using the apoptotic index is shown in Figure 2B.
Exposure to near infrared light destroyed cancer cells with high affinity to CS-GGS nanoparticles in vitro

We evaluated death in cells induced by loading CS-GGS nanoparticles and exposure to near infrared light in vitro. Three types of epithelial cells (HET-1A, benign; BAR-T, metaplasia; OE-19, adenocarcinoma) were treated with CSGGS nanoparticles for 24 hours, and then exposed to near infrared light. We found that the OE-19 cells showed very high affinity for the nanoparticles, and most OE-19 cells loaded with nanoparticles were completely ablated upon exposure to near infrared light. Under the microscope, most Het-1A cells did not show uptake of CS-GGS nanoparticles, whereas a few BAR-T cells showed a small amount of uptake compared with OE-19 cells (Figure 3). The BAR-T cells did not show any uptake of nanoparticles, with most of the nanoparticles remaining clustered in the extracellular regions. Exposure to near infrared light destroyed most of the OE-19 cells, whereas the HET-1A and BAR-T cells remained viable (Figure 3).

\section{Effects of CS-GGS nanoparticles and near infrared light on benign, metaplastic, and cancer cells}

We demonstrated that near infrared light could destroy the cells loaded with gold nanoparticles but not the control cells. This observation potentially reflects a photothermal effect occurring when the gold nanoparticles are heated. We then evaluated the effects of the nanoparticles and near infrared light exposure on cell viability in vitro. To quantify cell viability in response to exposure to near infrared light and to the nanoparticles, we performed an MTT assay in which optical density was used as an index for cell growth. As shown in Figure 4A, neither treatment with CS-GGS nanoparticles nor exposure to near infrared light affected the viability of any of the three cell lines after 24 hours when compared with controls $(P>0.05)$. However, the cell death rate was increased significantly in OE-19 cells loaded with the nanoparticles after exposure to near infrared light (Figure 4A). In addition, we evaluated whether gold nanoparticles and exposure to near infrared light could affect expression of cell defense proteins (manganese superoxide dismutase, catalase, and copper/zinc superoxide dismutase) or the cell death effector (caspase-3). These parameters were chosen because a large body of work implicates mitochondrial impairment as being an important cause of cell death, particularly given that the mitochondria serve 


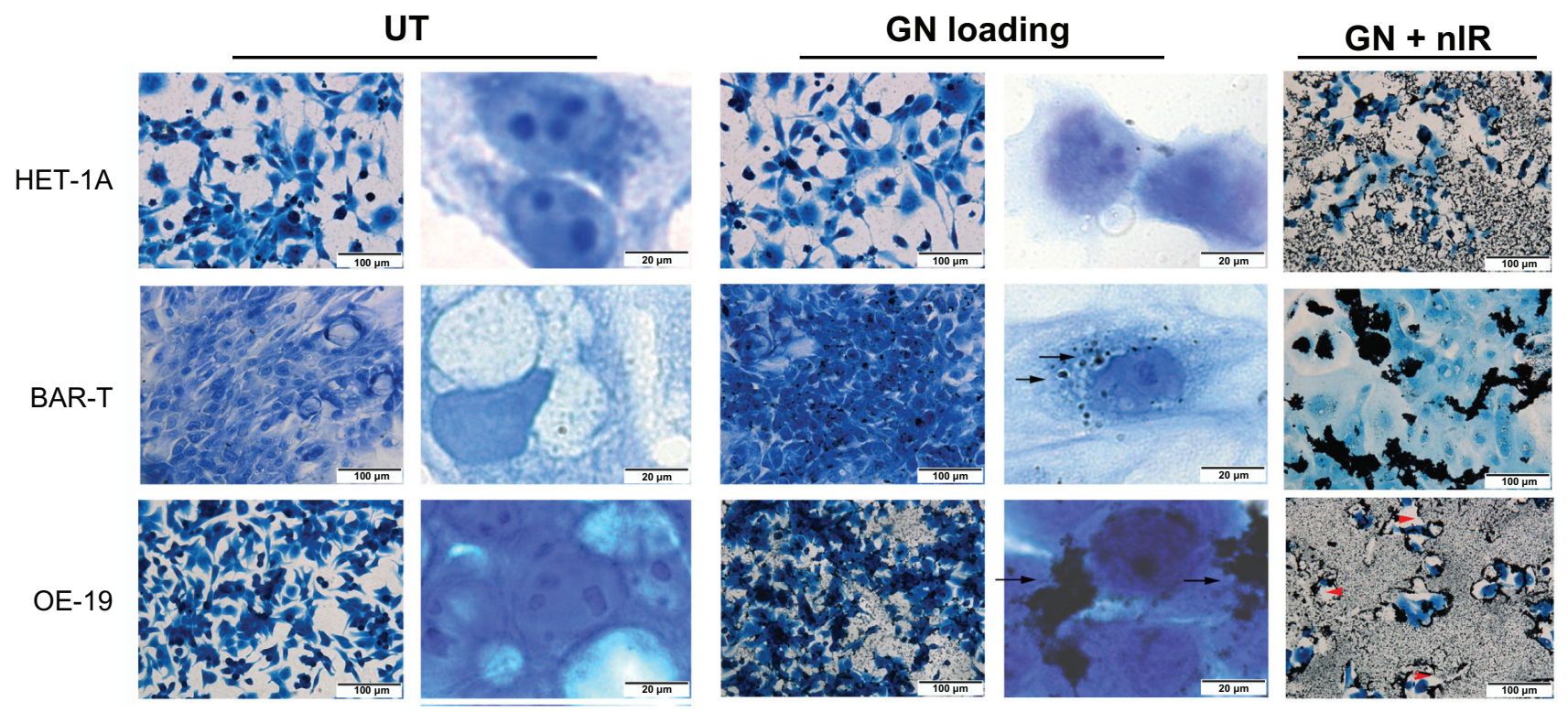

Figure 3 Cytotoxic effect of loading with gold nanoparticles in three esophageal cell lines (first column is lower magnification and second column is higher magnification). Notes: Arrow: gold nanoparticle loading in cells. Red arrow head: cells were removed after near infrared light exposure.

Abbreviations: UT, untreated; GN, gold nanoparticles; GN + nIR, gold nanoparticles treated with infrared light.

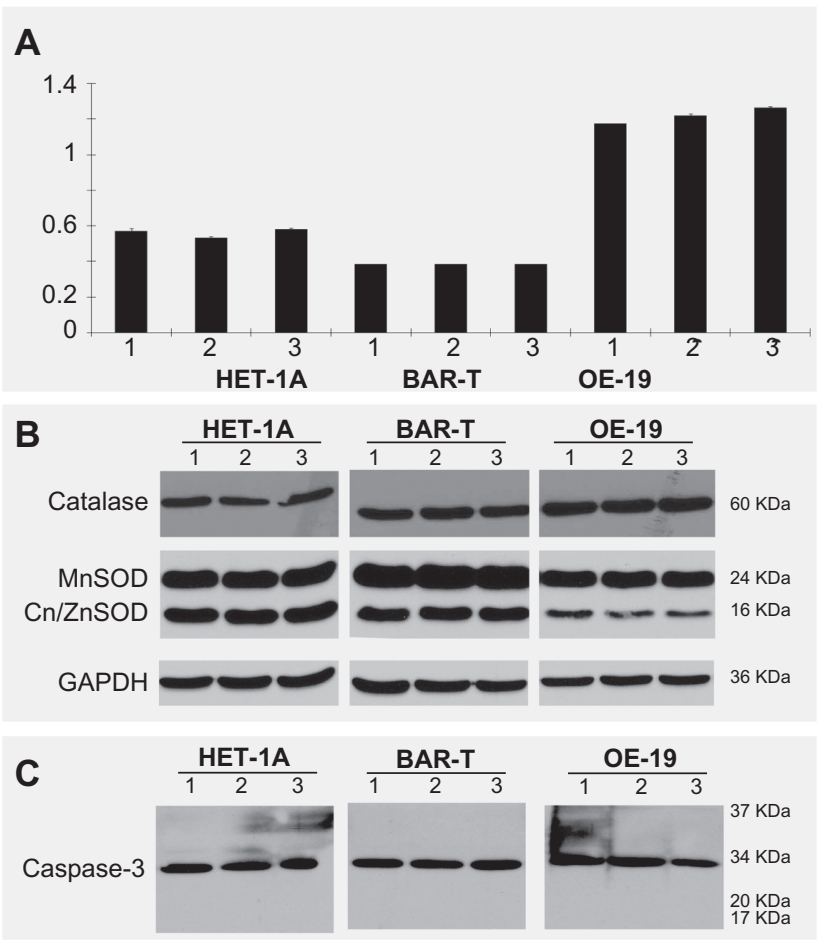

Figure 4 Effects of gold nanoparticle loading and exposure to near infrared light in three esophageal cell lines. (A) Effect of gold nanoparticles and near infrared light on cell viability in all three cell lines. There is no difference in cell viability after treatment with gold nanoparticles alone or exposure to near infrared light alone. (B) The effect of gold nanoparticles and nIR light on MnSOD, Cu/ZnSOD and catalase in three cell lines. There is no difference for the key protective proteins (catalase, MnSOD, Cu/ZnSOD, and GAPDH) in three cell lines. (C) The effect of gold nanoparticles and near infrared light on caspase- 3 in all three cell lines.

Notes: Caspase- 3 expression is in an inactive form in all three cell lines and no active forms, such as p20 or pl7, were cleaved in the three cell lines after treatment with gold nanoparticles or exposure to near infrared light. as gatekeepers for the "life-or-death" decision. ${ }^{36}$ Therefore, we studied mitochondrial manganese superoxide dismutase, which is a major cell defense protein against cell death. The other two major proteins, catalase and copper/zinc superoxide dismutase, are also important in cell defense against insults from oxidative stress. Caspase- 3 is an important cell death effector in the extrinsic and mitochondrial pathways. We evaluated the responses of all these proteins to treatment with CS-GGS nanoparticles and exposure to near infrared light in the three esophageal cell lines. The Western blot results show that neither the nanoparticles nor exposure to near infrared light affected these proteins (Figure 4B and C).

\section{Discussion}

Current multidisciplinary treatments for esophageal adenocarcinoma include radiotherapy, chemotherapy, and surgical resection. However, there is still a need to optimize local therapy using novel alternatives which overcome toxicity and intolerance and improve the present modest response rates. In this study, we investigated the possibility of treating esophageal cancer by introducing near infrared-absorbing CS-GGS nanoparticles and laser exposure through an endoscope in our established rat EDA model. The CS-GGS nanoparticles were taken up readily by tumor cells, with relatively low cytotoxicity to healthy cells. This novel material has many attractive features, including biocompatibility, no heavy metal 
toxicity, and strong optical absorption in the near infrared region, allowing development for photothermal therapeutic application.

This study demonstrates three advantages of a potential photoablative therapy to combat esophageal cancer:

- endoscopic delivery, whereby gold nanoparticles can be delivered with the assistance of a microendoscope via a needle and protective sheath; near infrared light exposure is successfully performed using a microfiber probe integrated with a laser system in the esophageal lumen; and endoscopic delivery may conceivably provide the opportunity to ablate esophageal cancer in patients who are not candidates for surgery

- two techniques which are each innocuous until combined, with near infrared light exposure not affecting normal esophageal tissue unless it was loaded with CS-GGS nanoparticles, suggesting that gold nanoparticles coupled with near infrared light exposure could enable thermalbased ablation targeted specifically at cancerous tissue, which is consistent with other reports ${ }^{14}$

- an ability to target cancer cells selectively, as indicated in vitro by the high affinity of malignant OE-19 cells for CS-GGS nanoparticles, whereas the two benign cell lines, ie, BAR-T and HET-1A cells, showed less affinity; the differential uptake of CS-GGS between cancerous and benign tissue could enable targeted destruction of only cancerous tissue.

There are questions that remain to be answered, as well as technical obstacles to be overcome in future studies. First, loading with CS-GGS nanoparticles could be a dynamic process, in which accumulation of the nanoparticles in esophageal adenocarcinoma could reach a peak at a certain time point in vivo. Peak loading could involve an "optimal therapeutic window" for near infrared light exposure, and this will need to be explored in future research. Second, it needs to be confirmed that normal esophageal epithelial or submucosal cells do not take up CS-GGS nanoparticles in vivo, to ensure that near infrared light only destroys cancer cells loaded with nanoparticles and not normal healthy cells. In our study, we found that some regions on the esophageal mucosa were "burned" on exposure to near infrared light, and these regions were later demonstrated to include both cancerous and adjacent healthy tissue. In the in vitro study, we have found that HET-1A, a benign esophageal cell line, had very low affinity for gold nanoparticles, so the observed photothermal ablation effect on adjacent benign tissue could indicate infiltration by inflammatory cells, such as phagocytes, but this needs to be confirmed.
Therefore, uptake of these nanoparticles in both cancerous and benign tissue needs to be evaluated further in vivo. A dynamic uptake study may help us to understand better the nature of nanoparticle loading in cancerous tissue and to what extent, if any, this occurs in benign esophageal mucosa. A dynamic study of this sort would allow us to determine if nanoparticle loading differs between benign and cancerous tissue, and if there is a time point when the nanoparticles become highly concentrated in cancerous tissue but not in healthy tissue. Third, it is generally accepted that these nanoparticles are not associated with heavy metal toxicity. We did not observe any toxic effects of CS-GSS nanoparticles on the benign HET-1A and BAR-T cell lines. However, the potential for metabolism of CS-GSS nanoparticles in the body needs to be evaluated further in vivo, particularly with regard to any toxic effects on the brain, kidney, and heart.

Regarding the technical obstacles, there are some important issues which require attention in a future study. In our study, the CS-GSS nanoparticles were sprayed onto the surface of suspicious mucosa at a high concentration (50 OD) to ensure absorption. One needs to take great care with direct injection of nanoparticles so as to minimize the risk of them becoming trapped in the interstitium of benign tissue. Our in vitro study showed that although the benign Het-1A cells did not become loaded with nanoparticles, we did find a large amount of gold nanoparticles attached on the bottom of the culture plate, which was probably the result of precoating the plate with a mixture of fibronectin, collagen type I, and bovine serum albumin, which are necessary for the growth of Het-1A cells. These extracellular matrices are very rich in interstitium, so loading of esophageal tissue with CS-GSS nanoparticles needs to be well controlled.

Another important issue is the use of a laser probe in the esophageal lumen. We used a murine endoscope to guide cannulation of a microfiber probe to the target esophageal epithelium. Although exposure to near infrared light is safe in esophageal epithelium not loaded with nanoparticles, the microfiber optic probe could cause injury to this tissue if it is too close to is directly touching the surface of the esophageal epithelium. During an attempt at external near infrared illumination, in which we placed the laser directly on top of the body, no ablation of the esophageal mucosa was observed, even though the mucosa was loaded with nanoparticles. Part of the reason for this could have been the depth of penetration of near infrared light into the body. A modified radiofrequency might need to be used to improve penetration of near infrared light into deeper sites of interest. 
In this study, we investigated the ability of near infraredabsorbing CS-GGS nanoparticles to destroy esophageal cancer cells using an endoscopic procedure. Taken together, our study shows that introducing near infrared-absorbing CS-GGS nanoparticles into the esophageal mucosa to ablate cancer tissue under endoscopy is technically feasible. This technology holds exciting potential for use in patients with esophageal cancer who are not candidates for surgery, although considerable research on this strategy remains to be done in the future.

\section{Acknowledgment}

This work was supported by The Clinical and Translational Science Pilot Grant Program's Basic Award and Innovative Award at University of Louisville 2010, and by the Coulter Foundation Clinical Translational Research Award. BAR-T is a human hTERT-immortalized benign Barrett's cell line, which was generously gifted by Rhonda Souza and SJ Spechler at the Department of Medicine, VA North Texas Health Care System and the University of Texas Southwestern Medical School, Dallas, TX, USA.

\section{Disclosure}

None of the authors has a commercial or institutional association, currently or within the past five years, that might pose a potential, perceived, or real conflict of interest in this work.

\section{References}

1. Jemal A, Siegel R, Ward E, Hao Y, Xu J, Thun MJ. Cancer statistics, 2009. CA Cancer J Clin. 2009;59:225-249.

2. Lindblad M, Ye W, Lindgren A, Lagergren J. Disparities in the classification of esophageal and cardia adenocarcinomas and their influence on reported incidence rates. Ann Surg. 2006;243:479-485.

3. Vizcaino AP, Moreno V, Lambert R, Parkin DM. Time trends incidence of both major histologic types of esophageal carcinomas in selected countries, 1973-1995. Int J Cancer. 2002;99:860-868.

4. Altorki NK, Oliveria S, Schrump DS. Epidemiology and molecular biology of Barrett's adenocarcinoma. Semin Surg Oncol. 1997;13:270-280.

5. Blot WJ, McLaughlin JK. The changing epidemiology of esophageal cancer. Semin Oncol. 1999;26:2-8.

6. Farrow DC, Vaughan TL. Determinants of survival following the diagnosis of esophageal adenocarcinoma (United States). Cancer Causes Control. 1996;7:322-327.

7. Ku GY, Ilson DH. Role of neoadjuvant therapy for esophageal adenocarcinoma. Surg Oncol Clin N Am. 2009;18:533-546.

8. Lawson JD, Otto K, Grist W, Johnstone PA. Frequency of esophageal stenosis after simultaneous modulated accelerated radiation therapy and chemotherapy for head and neck cancer. Am J Otolaryngol. 2008;29: 13-19.

9. Boca SC, Potara M, Gabudean AM, Juhem A, Baldeck PL, Astilean S. Chitosan-coated triangular silver nanoparticles as a novel class of biocompatible, highly effective photothermal transducers for in vitro cancer cell therapy. Cancer Lett. 2011;311:131-140.
10. Moghimi SM. Recent developments in polymeric nanoparticle engineering and their applications in experimental and clinical oncology. Anticancer Agents Med Chem. 2006;6:553-561.

11. Moon GD, Choi SW, Cai X, et al. A new theranostic system based on gold nanocages and phase-change materials with unique features for photoacoustic imaging and controlled release. J Am Chem Soc. 2011; 133:4762-4765.

12. Moghimi SM, Kissel T. Particulate nanomedicines. Adv Drug Deliv Rev. 2006;58:1451-1455.

13. Avedisian CT, Cavicchi RE, McEuen PL, Zhou X. Nanoparticles for cancer treatment: role of heat transfer. Ann N Y Acad Sci. 2009;1161: $62-73$.

14. Cardinal J, Klune JR, Chory E, et al. Noninvasive radiofrequency ablation of cancer targeted by gold nanoparticles. Surgery. 2008;144: 125-132.

15. Gentili D, Ori G, Comes FM. Double phase transfer of gold nanorods for surface functionalization and entrapment into PEG-based nanocarriers. Chem Commun (Camb). 2009;39:5874-5876.

16. Takahashi H, Niidome Y, Niidome T, Kaneko K, Kawasaki H, Yamada S. Modification of gold nanorods using phosphatidylcholine to reduce cytotoxicity. Langmuir. 2006;22:2-5.

17. Niidome T, Yamagata M, Okamoto Y, et al. PEG-modified gold nanorods with a stealth character for in vivo applications. J Control Release. 2006; 114:343-347.

18. Huang X, El Sayed IH, Qian W, El Sayed MA. Cancer cell imaging and photothermal therapy in the near-infrared region by using gold nanorods. J Am Chem Soc. 2006;128:2115-2120.

19. Chen J, Wang D, Xi J, et al. Immuno gold nanocages with tailored optical properties for targeted photothermal destruction of cancer cells. Nano Lett. 2007;7:1318-1322.

20. Gobin AM, Lee MH, Halas NJ, James WD, Drezek RA, West JL. Near-infrared resonant nanoshells for combined optical imaging and photothermal cancer therapy. Nano Lett. 2007;7:1929-1934.

21. Lowery AR, Gobin AM, Day ES, Halas NJ, West JL. Immunonanoshells for targeted photothermal ablation of tumor cells. Int J Nanomedicine. 2006;1:149-154.

22. O'Neal DP, Hirsch LR, Halas NJ, Payne JD, West JL. Photo-thermal tumor ablation in mice using near infrared-absorbing nanoparticles. Cancer Lett. 2004;209:171-176.

23. Hirsch LR, Stafford RJ, Bankson JA, et al. Nanoshell-mediated nearinfrared thermal therapy of tumors under magnetic resonance guidance. Proc Natl Acad Sci U S A. 2003;100:13549-13554.

24. Day ES, Bickford LR, Slater JH, Riggall NS, Drezek RA, West JL. Antibody-conjugated gold-gold sulfide nanoparticles as multifunctional agents for imaging and therapy of breast cancer. Int J Nanomedicine. 2010;5:445-454.

25. Gobin AM, Watkins EM, Quevedo E, Colvin VL, West JL. Nearinfrared-resonant gold/gold sulfide nanoparticles as a photothermal cancer therapeutic agent. Small. 2010;6:745-752.

26. Gobin AM, O'Neal DP, Watkins DM, Halas NJ, Drezek RA, West JL. Near infrared laser-tissue welding using nanoshells as an exogenous absorber. Lasers Surg Med. 2005;37:123-129.

27. Gobin AM, Moon JJ, West JL. EphrinA I-targeted nanoshells for photothermal ablation of prostate cancer cells. Int J Nanomedicine. 2008;3:351-358.

28. Li Y, Wo JM, Su RR, Ray MB, Martin RC. Loss of manganese superoxide dismutase expression and activity in rat esophagus with external esophageal perfusion. Surgery. 2007;141:359-367.

39. Martin RC, Liu Q, Wo JM, Ray MB, Li Y. Chemoprevention of carcinogenic progression to esophageal adenocarcinoma by the manganese superoxide dismutase supplementation. Clin Cancer Res. 2007;13: $5176-5182$.

30. Woodall CE, Li Y, Liu QH, Wo J, Martin RC. Chemoprevention of metaplasia initiation and carcinogenic progression to esophageal adenocarcinoma by resveratrol supplementation. Anticancer Drugs. 2009;20:437-443 
31. Li Y, Woodall C, Wo JM, et al. The use of dynamic positron emission tomography imaging for evaluating the carcinogenic progression of intestinal metaplasia to esophageal adenocarcinoma. Cancer Invest. 2008;26:278-285.

32. Schiffman SC, Li Y, Dryden G, Li X, Martin RC. Positive correlation of image analysis by mini-endoscopy with micro-PET scan and histology in rats after esophagoduodenal anastomosis. Surg Endosc. 2010;24: 2835-2841.

33. Ismail-Beigi F, Horton PF, Pope CE. Histological consequences of gastroesophageal reflux in man. Gastroenterology. 1970;58:163-174.
34. Li Y, Wo JM, Su RR, Ray MB, Jones W, Martin RC. Esophageal injury with external esophageal perfusion. J Surg Res. 2005;129:107-113.

35. Li Y, Wo JM, Ellis S, Ray MB, Jones W, Martin RC. A novel external esophageal perfusion model for reflux esophageal injury. Dig Dis Sci. 2006;51:527-532.

36. Fosslien E. Mitochondrial medicine-molecular pathology of defective oxidative phosphorylation. Ann Clin Lab Sci. 2001;31:25-67.
International Journal of Nanomedicine

\section{Publish your work in this journal}

The International Journal of Nanomedicine is an international, peerreviewed journal focusing on the application of nanotechnology in diagnostics, therapeutics, and drug delivery systems throughout the biomedical field. This journal is indexed on PubMed Central,

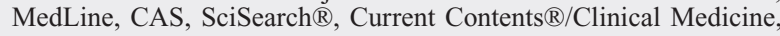

\section{Dovepress}

Journal Citation Reports/Science Edition, EMBase, Scopus and the Elsevier Bibliographic databases. The manuscript management system is completely online and includes a very quick and fair peer-review system, which is all easy to use. Visit http://www.dovepress.com/ testimonials.php to read real quotes from published authors.

Submit your manuscript here: http://www.dovepress.com/international-journal-of-nanomedicine-journal 